\title{
Mehrkanaliges Feedback in Bachelorseminaren
}

In diesem schreibintensiven Seminar wurden vielfältige Feedback (FB)-Mechanismen implementiert. Dabei wurden drei Faktoren besonders berücksichtigt: die Qualität des FB (das FB soll zu einem konstruktiven Lernfortschritt führen), die Quantität und Diversität des FB (FB aus unterschiedlichen Quellen und in verschiedenen Formen trägt eher zu einem ganzheitlichen Lernfortschritt bei; die Priorisierung des Feedbacks wird unterstützt, da wichtige Punkte eher wiederholt genannt werden) und Nachhaltigkeit des FB (die zeitlichen Ressourcen des LV-Leiters sind beschränkt).

Unter diesen Gesichtspunkten wurde FB als wichtigste Lernressource für dieses Seminar definiert und das in Abbildung 1 dargestellte FB-System installiert.

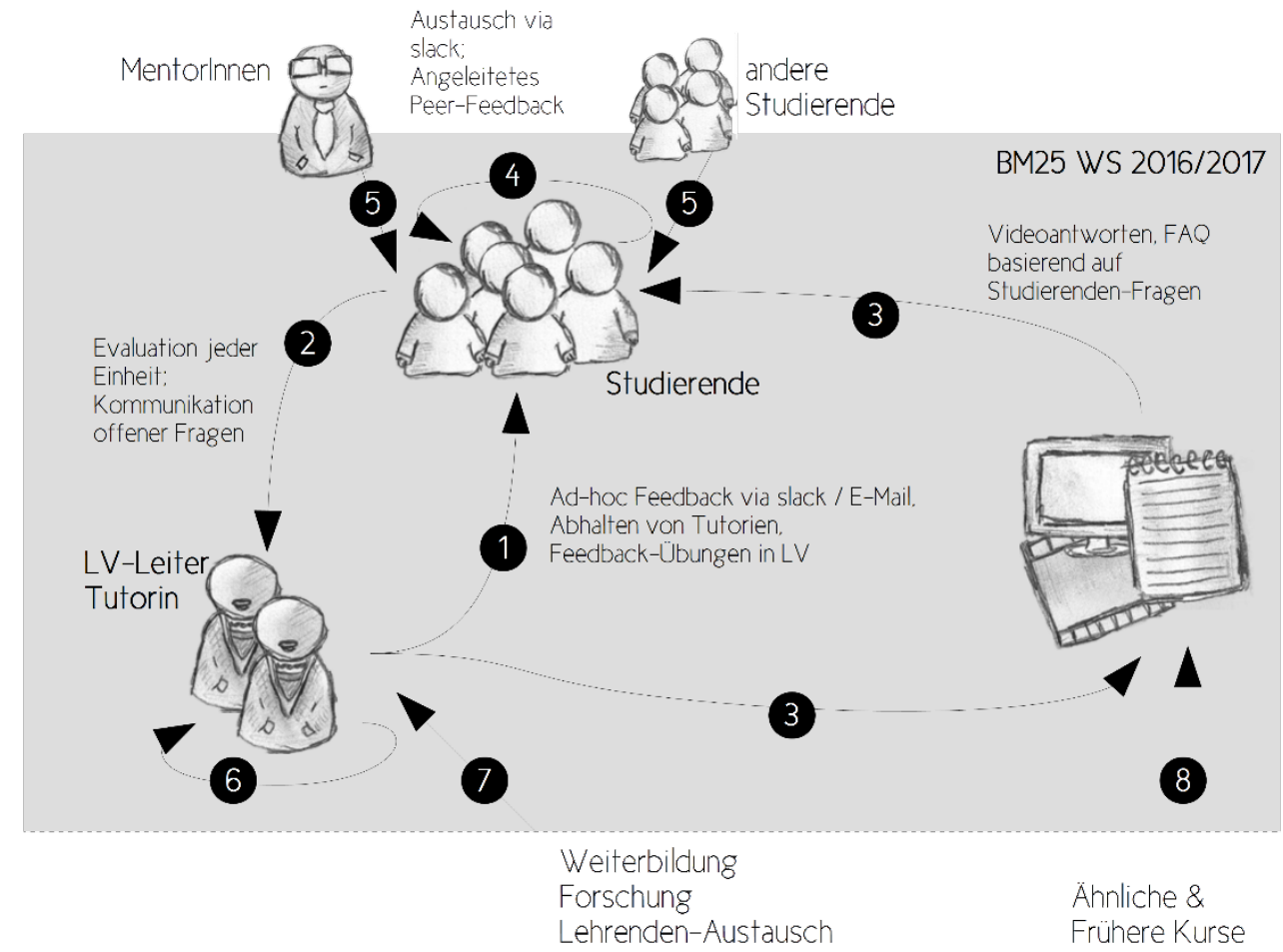

Abbildung 1: Übersicht über die Feedback-Mechanismen im Seminar.

An dieser Stelle sollen nur die Besonderheiten dieses Seminars hervorgehoben werden:

Die Studierenden gaben jede LV-Einheit schriftliches FB an den LV-Leiter (siehe 2 in Abbildung 1): Was läuft gut? Wo bestehen Herausforderungen? Dieses FB führt zu einer Anpassung des LVVerlaufs; häufig auftretende Fragen/Probleme wurden per Video auf Moodle beantwortet (formatives Gruppenfeedback). Diese Inhalte können für die nächsten LVs mitverwendet werden, was dem Gebot der Effizienz entgegen kommt (3).

In der LV wurde die verwendete Technologie dem LV-Verlauf angepasst: „Langsame“, formalere Kommunikation erfolgte via Moodle (z.B. Videos) oder E-Mail (24-Stunden Antwort-Policy), informellere, schnellere (Peer-)Feedback-Ansuchen konnten in einer slack ${ }^{1}$-Gruppe gestellt werden $(\mathbf{4}, \mathbf{5})$. Statt der Tutorien wurde über die Weihnachtsferien ein virtuelles Treffen via Google hangouts ${ }^{2}$ für individuelles FB vom LV-Leiter angeboten ((0).

Die Studierenden kamen durch die slack-Gruppe in den Austausch mit Studierenden anderer Institutionen ${ }^{3}$ und (höhersemestriger) „MentorInnen“. Der (informelle) FB -Austausch erfolgte in beide Richtungen. Dies trug zur Sicherung der Diversität des FB bei.

Vielfältige Übungen zum Feedback-Geben (und -Nehmen) wurden durchgeführt. Hervorzuheben ist ein dem wissenschaftlichen Publikationsprozess nachempfundener Peer-Review Prozess (siehe Abbildung 2). Dieser Peer-Feedback Prozess wurde laufend evaluiert um eine hohe Qualität zu gewährleisten. Eine weitere Übung war zum Beispiel eine Poster-Messe, bei der verbales Peer- und LV-Leiter-Feedback gegeben wurde. Es wurde auch einer Schreibmentorin des CTL viel Raum gegeben, Feedback-Übungen durchzuführen. Die Peer-Feedback-Übungen waren über den Verlauf

\footnotetext{
${ }^{1}$ https://www.slack.com/

2 https://hangouts.google.com/

${ }^{3}$ Zu dieser Gruppe werden alle Bachelor und Master Studierenden eingeladen, die Kurse zu wissenschaftlichem Arbeiten unter meiner Leitung besuchen oder besuchten (Universität Wien, FHWien der WKW, Universität Maastricht).
} 
des Seminars gesehen so gestaltet, dass der Freiraum, den die Studierenden hatten, zunahm: In den ersten Übungen stehen noch klare Bewertungskriterien bzw. Feedback-Leitfragen zur Verfügung (angeleitetes Peer-Feedback), später können die Studierende eine individuellere Perspektive entwickeln (vgl. „scaffolding“).

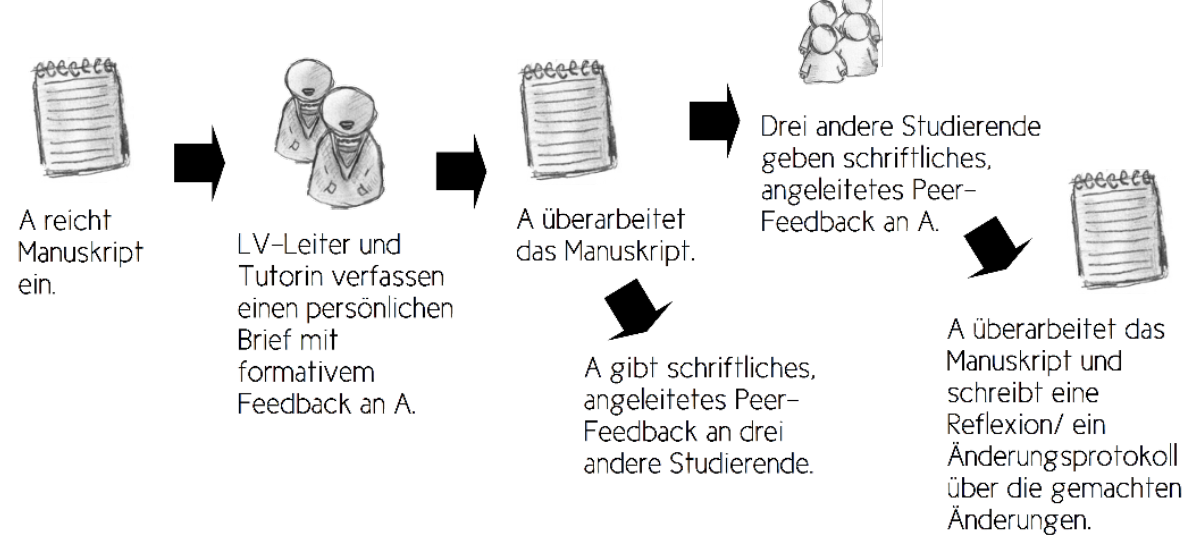

Abbildung 2: Beispielhafter Peer-Feedbackprozess im Rahmen einer Übung.

Studierende, die das Seminar mit einem „Sehr Gut" abgeschlossen haben, wurde eine gemeinsame Publikation in einer wissenschaftlichen Zeitschrift basierend auf der Arbeit angeboten (wenn auch andere Kriterien wie z.B. Neuheit erfüllt waren). Im Rahmen dieser Umarbeitung kann der LV-Leiter ein wesentlich ausführlicheres formatives FB aus Ko-Autorenrolle geben, dass die Aufgabenstellung der eigentlichen Arbeit weit übererfüllt. Eine Referenz einer früheren Studentin belegt die Sinnhaftigkeit dieses Angebots aus Perspektive der Lernenden. 